\title{
Treatment of migraine attacks based on the interaction with the trigemino-cerebrovascular system
}

\author{
Andrea Stephanie Link • Anikó Kuris • \\ Lars Edvinsson
}

Received: 2 October 2007 / Accepted: 28 November 2007 / Published online: 23 January 2008

(C) Springer-Verlag 2008

\begin{abstract}
Primary headaches such as migraine are among the most prevalent neurological disorders, affecting up to one-fifth of the adult population. The scientific work in the last decade has unraveled much of the pathophysiological background of migraine, which is now considered to be a neurovascular disorder. It has been discovered that the trigemino-cerebrovascular system plays a key role in migraine headache pathophysiology by releasing the potent vasodilator calcitonin gene-related peptide (CGRP). This neuropeptide is released in parallel with the pain and its concentration correlates well with the intensity of the headache. The development of drugs of the triptan class has provided relief for the acute attacks but at the cost of, mainly cardiovascular, side effects. Thus, the intention to improve treatment led to the development of small CGRP receptor antagonists such as olcegepant (BIBN4096BS) and MK-0974 that alleviate the acute migraine attack without acute side events. The purpose of this review is to give a short overview of the pathological background of migraine headache and to illustrate the mechanisms behind the actions of triptans and the promising CGRP receptor blockers.
\end{abstract}

Keywords Trigemino-cerebrovascular system .

CGRP · Triptan · Olcegepant · MK-0974

\section{The trigemino-cerebrovascular system}

Pain-sensitive structures such as the intracranial vessels and the meninges, especially the dura mater, are supplied

A. S. Link · A. Kuris · L. Edvinsson ( $\square)$

Biomedical Center Lund, Lund, Sweden

e-mail: Lars.Edvinsson@med.lu.se with sensory nerve fibers. In fact they are innervated by the ophthalmic ramus of the first branch of the trigeminal nerve that arises from pseudounipolar neurons located in the trigeminal ganglion. Those neurons project onto second order sensory neurons in the trigmenial nucleus caudalis (TNC) in the brain stem and its related extensions down to the C2-level called the trigeminocervical complex [1]. From this region a signal is transmitted to the ventroposterior thalamus leading to activation in cortical areas, including frontal cortex, insulae and cingulate cortex. This results in the experience of pain (Fig. 1). In addition, a migraine active region has been pointed out in the brainstem by using positron emission tomography (PET) [2-4]. Thus, an involvement of the trigemino-cerebrovascular system in the transmission of nociceptive information to the central nervous system is a viable position.

\section{The trigemino-cerebrovascular reflex}

There is a dense supply of CGRP-containing nerve fibers originating in the first division of the trigeminal ganglion that innervate intracranial vessels. Experimental studies have shown that induction of vasoconstriction provoked rapid normalization of the vascular tone due to-in partreflex activation of the trigeminal nerve. Vasoconstriction triggers an antidromic release of sensory trigeminal neuropeptides, mainly CGRP, being a strong intracranial dilatator [5, 6]. After denervation there is no alteration in the contractile response of the vessel but the time to attain initial basal tone is markedly prolonged. This is of importance as headache may be initiated by a spreading wave of depression of cortical neurons resulting in contraction of some parts of the cerebral circulation. This phenomenon has now been shown in man [7] and could 


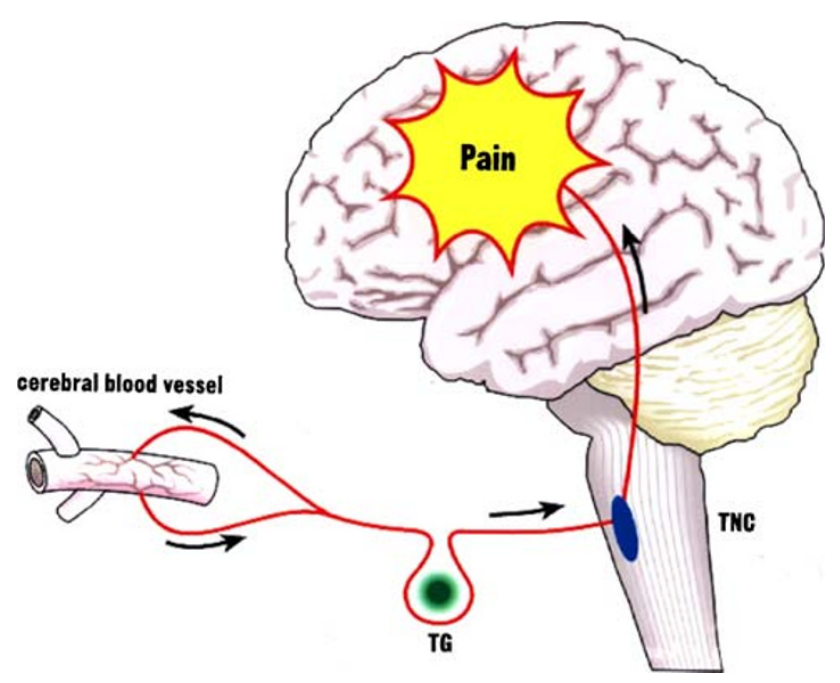

Fig. 1 In migraine attacks, sensory nerves, which innervate the cerebral blood vessels, are activated to antidromically release calcitonin gene-related peptide. They originate from the first branch of the trigeminal nerve that arises from pseudounipolar neurons located in the trigeminal ganglion $(T G)$. Those neurons project onto second order sensory neurons in the trigeminal nucleus caudalis $(T N C)$ from where a signal is transmitted to the cortex

activate the trigeminal vascular system to release CGRP to reestablish the vascular tone. Thus, the trigemino-cerebrovascular reflex is probably part of a counter-balancing system, which is able to mediate dilatation of brain vessels and sustain cerebral blood flow [8]. This may assure the maintenance of local brain blood flow within normal limits as the cerebral circulation requires high and constant flow. Activation of the neurons in the trigeminal ganglion leads on the other hand to an activation of neurons in the trigeminocervical complex that project to the central nervous system (CNS) and mediate the central aspects of pain $[1,9]$.

\section{Calcitonin gene-related peptide}

CGRP is a neuropeptide consisting of 37 amino acids and is predominantly expressed in the nervous system. It originates from the calcitonin gene encoding calcitonin and CGRP depending upon alternative splicing.

Histochemical studies of cerebral perivascular nerve fibers and human trigeminal ganglia revealed that almost half of the cell bodies contain CGRP [10, 11]. Although many other signal molecules are located in the trigeminal ganglion, CGRP is the most abundant in man [12] and can be seen as a marker for trigeminal activity. Furthermore it is the only transmitter reliably released in acute migraine attacks at present. There was a marked increase in CGRP levels during migraine attacks, but no changes could be observed for other neuropeptides such as vasoactive intestinal peptide (a marker for parasympathetic activity), neuropeptide $\mathrm{Y}$ (sympathetic activity) and substance $\mathrm{P}$ (sensory activity) when their levels were analyzed in the cranial venous outflow during migraine attacks $[8,13]$.

In migraine attacks elicited within clinical studies by administration of nitroglycerin a good correlation has been described between increased levels of CGRP and the intensity of the headache [14, 15]. But it is noteworthy to mention that nitroglycerin elicits head pain, but not always genuine migraine attacks. There may be a need for the subject to be in a "prone phase", where the trigeminocerebrovascular system might be hyper-reactive as it was demonstrated in cluster headache [16].

\section{Triptans}

The increased cranial venous levels of CGRP observed in conjunction with migraine attacks thus indicate activation of the trigeminal system. Administration of sumatriptanbelonging to the highly effective anti-migraine drugs called triptans or $5-\mathrm{HT}_{1 \mathrm{~B} / 1 \mathrm{D}}$ agonists-results in alleviation of the headache and normalization of the cranial venous CGRP levels [8]. They are thought to act mainly as powerful vasoconstrictors of cerebral vessels via $5-\mathrm{HT}_{1 \mathrm{~B}}$ receptors, which have been shown to be expressed in the medial smooth muscle cell layer of the human middle meningeal [17] and cerebral arteries [18].

In addition, triptans may bind to presynaptic $5-\mathrm{HT}_{1 \mathrm{~B} / 1 \mathrm{D}}$ receptors on trigeminal afferents to inhibit nerve activity and hence reduce CGRP release. 5- $\mathrm{HT}_{1 \mathrm{~B} / 1 \mathrm{D}}$ receptors were found on trigeminal sensory fibers [19], trigeminal ganglion cells [20] and in the trigemino-cerebrovascular system in man [21]. Furthermore, the $5-\mathrm{HT}_{1 \mathrm{~B} / 1 \mathrm{D}}$ receptors were co-localized with CGRP in the human trigeminal ganglion [20].

Autoradiographic studies using labeled zolmitriptan confirmed the presence of $5-\mathrm{HT}_{1 \mathrm{~B} / 1 \mathrm{D}}$ receptors in certain brain stem nuclei that are involved in cranial nociceptive processing [22] and are likely to be activated during migraine.

Thus, it can be presumed that a potential relaxation of intracranial blood vessels during migraine attacks can be normalized by triptans, which activate the cerebrovascular $5-\mathrm{HT}_{1 \mathrm{~B}}$ receptors and thereby cause vasoconstriction. By stimulating $5-\mathrm{HT}_{1 \mathrm{~B} / 1 \mathrm{D}}$ receptors, triptans inhibit the release of CGRP from the perivascular nerve terminals and the trigeminal ganglion cells. Activation of $5-\mathrm{HT}_{1 \mathrm{~B} / 1 \mathrm{D}}$ receptors at the level of the CNS (trigeminal nucleus caudalis) may interrupt central aspects of the headache process.

Recently the relationship between pharmacokinetic and pharmacodynamic aspects of triptans in migraine was investigated. It has been observed that subcutaneous 
administration of sumatriptan is more effective than oral application. The difference in efficacy is most likely due to the rapid initial rise in plasma level after subcutaneous administration [23]. Furthermore migraine attacks may influence gastric stasis leading to delayed absorption of drugs $[24,25]$. Thus, to increase efficacy of treatment, attention should be paid to the development of migraine drugs, which can be quickly absorbed.

Triptans cause sometimes cardiovascular adverse effects as they also act as constrictors in coronary arteries. But triptans are considered as more powerful vasoconstrictors in human isolated cerebral arteries than in coronary arteries. They are at least threefold more potent [26]. The reason for the higher potency and efficiency of triptans in intracranial arteries could be due to at least three differences. First there is a higher number of $5-\mathrm{HT}_{1 \mathrm{~B}}$ receptors in those vessels compared to peripheral arteries [18, 27]. Secondly there are few, if any, $5-\mathrm{HT}_{2 \mathrm{~A}}$ receptors in intracranial vessels whereas the $5-\mathrm{HT}_{2 \mathrm{~A}}$ receptor population dominates in coronary arteries [27]. Thirdly, there might be potential differences in coupling of the 5- $\mathrm{HT}_{1 \mathrm{~B}}$ receptors between cerebral and coronary arteries. In consideration of the fact that triptans are weak constrictors in coronary arteries [28, 29] and have little impact on coronary blood flow, the clinical consequences in healthy subjects are minor. However, small contractions may anyhow have proportionally greater impact in diseased artery with luminal narrowing. Triptans are hence contra-indicated in patients with known cardiovascular disease.

\section{Sensitization in migraine}

Treatment with triptans achieves good results in patients suffering from sensitization. Sensitization is defined as an increased afferent activity in response to an unchanged stimulus [30] and both peripheral and central sensitization may play a role in migraine attacks. Peripheral sensitization of meningeal perivascular nociceptors arising from trigeminal neurons can clinically explain phenomena like intracranial hypersensitivity (worsening pain during physical activity) and the throbbing element in migraine pain. This may be due to normally innocuous meningeal nociceptors becoming hyper-responsive to rhythmic fluctuation in intracranial pressure that is produced by normal arterial pulsations [31, 32].

Central sensitization of nociceptive second order neurons in the trigeminocervical complex can be the reason for extracranial tenderness and cutaneous allodynia. The latter is an abnormal sensory state in which otherwise innocuous stimuli are sensed as painful [31].

Two-thirds of patients develop allodynia during or even after a migraine attack [33]. Based on clinical studies new guidelines for triptan treatment in migraine has been established. They include that allodynia-free patients can receive good results from triptan treatment at any time during the migraine attack. Furthermore, patients suffering from allodynia can expect excellent results from triptans before allodynia gets established. Therefore they should take triptans as early as possible during the attack. After the onset of allodynia, migraineurs can still benefit from triptans, but pain relief may be incomplete [34-36].

However, a slightly different view has been expressed in a recent study [37]. After subcutaneous administration of sumatriptan, there was no difference in alleviation of migraine pain irrespective if the drug was given early or late. Therefore, it was suggested that the sensitization is of minor importance and that the relatively poor effect of sumatriptan late in migraine attacks is primarily due to gastric stasis [37].

\section{CGRP receptors}

Local vasodilatation and release of CGRP from the vascular nerve fiber endings-caused by electrical field stimulation or capsaicin treatment $[38,39]$-are attenuated by administration of triptans, but also by application of CGRP receptor blockers [40]. The proof that CGRP plays a key role in migraine was provided by demonstrating that the potent CGRP receptor antagonist olcegepant (BIBN4096) could abort acute migraine attacks to a comparable degree as sumatriptan $[41,42]$. In addition, CGRP receptors are located at several sites, which play an important role during migraine, including the cerebrovasculature [43-45], the trigeminocervical complex within the brainstem [46] and the trigeminal ganglion [47].

Functional CGRP receptors consist of three components, namely a seven-transmembrane domain G-protein coupled receptor called calcitonin receptor-like receptor (CLR), a receptor activity-modifying protein (RAMP) and a receptor component protein (RCP). CLR shares 55\% sequence identity with the calcitonin receptor. Co-expression of CLR with RAMP1 composes the CGRP receptor, while coexpression of CLR with either RAMP2 or RAMP3 represents the adrenomedullin (AM) receptor or possibly a combined receptor (CGRP and AM). Apart from contributing to the receptor specificity, RAMP1 is required for glycosylation and transport of CLR to the plasma membrane [48-50]. In addition, RAMP1 seems to be functionally rate limiting for CGRP receptor activity in the trigeminovascular system. It was proposed that elevated RAMP1 expression could sensitize the trigeminal ganglion of individuals to CGRP actions such as CGRP release and neurogenic inflammation [47].

The third component of the CGRP receptor, the accessory protein $\mathrm{RCP}$, is required for proper biological function 
as it is involved in coupling the receptor to downstream signaling pathways like the protein kinase A (PKA) pathway [51]. Activation of CGRP receptors in cultured trigeminal ganglion cells increased the cAMP levels, which in turn led to elevated CGRP promoter activity and increased CGRP mRNA levels. These results point to an autoactivation of CGRP expression and indicate that elevated release of CGRP in the trigeminal system may create a self-sustaining feedback loop [47].

\section{CGRP receptor antagonists olcegepant and MK-0974}

The excellent correlation between CGRP release and migraine headache has long pointed towards the potential usefulness of a specific CGRP receptor antagonist in the treatment of primary headaches [9, 52]. Thus, a series of small molecule CGRP receptor blockers were developed. Among those is olcegepant, which demonstrates extremely high affinity for human CGRP receptors, even higher affinity than the endogenous ligand [53]. Therefore it was selected for further pharmacological investigations. Olcegepant was shown to be a pure antagonist for the human CGRP receptor and to be selective against a broad panel of enzymes and receptors such as calcitonin, amylin or adrenomedullin receptors [54].

In isolated human temporal, middle meningeal and cerebral arteries olcegepant effectively inhibits CGRP induced dilatation $[44,55]$. That may be due to the block of the CGRP receptor as mRNA for CLR and RAMP1-3 has been found in human middle meningeal arteries, cerebral arteries as well as in microvessels [56] and CGRP receptor components could be detected in the human cerebral vasculature [43]. In addition, olcegepant dose-dependently inhibited vasodilatation induced by electrical stimulation of the trigeminal ganglion in primates [54].

One major advantage of a CGRP receptor blocker is the lack of vasoconstrictor ability. But blockade of the receptor of a strong vasodilatation involves a theoretical risk of causing both peripheral and cerebral vasoconstriction, especially as high affinity binding sites for CGRP have been reported in the heart, liver, spleen, intestine, urogenital system, skeletal muscles, and the skin [57-59]. But olcegepant in doses up to $10 \mathrm{mg}$ did not cause any clinical relevant changes of vital signs like blood pressure and pulse rate, in the ECG or in forearm blood flow in healthy volunteers [60]. Detailed studies of peripheral arteries such as mesenteric and coronary arteries $[44,61]$ revealed that those arteries show weaker responses to CGRP and lower degree of antagonism by olcegepant. This is possibly related to a lower number of CGRP receptors. In addition, a study with seven healthy volunteers disclosed that olcegepant had no influence on cerebral blood flow or on the blood flow velocity in the middle cerebral artery [62]. It was concluded that CGRP receptor blockade has no effect on the cerebral circulation in man.

However, it is still not clear where olcegepant acts as a CGRP antagonist. It has been reported to inhibit (1) dilatation of dural arteries initiated by CGRP [63], (2) CGRPinduced hypotension [40], and (3) increase in facial blood flow after trigeminal ganglion stimulation [54]. In contrast, olcegepant did not significantly inhibit changes in tone of cerebral arterioles or of local cortical cerebral blood flow [40]. Those results indicate that olcegepant poorly penetrates the blood-brain barrier, but is very effective in preventing vasodilatation of vessels without this feature [64]. Thus, this CGRP receptor blocker was suggested to exert its antagonistic effects mainly extracerebral, which correlates with results from a study of healthy volunteers [65]. In contrast, it was recently shown that CGRP-induced relaxation of the rat middle cerebral artery can only be inhibited by abluminally administered olcegepant as olcegepant was prevented from reaching the CGRP receptors by the arterial endothelium [66]. These results suggest that the anti-migraine effect of this CGRP receptor blocker is on the abluminal side of the blood-brain barrier. This is supported by the fact that olcegepant only has an antimigraine effect in high doses.

Anyhow, olcegepant was effective in a dose of $2.5 \mathrm{mg}$ in the treatment of acute migraine attacks [41]. The overall treatment effect revealed a responder rate (a moderate to severe headache going to mild or no headache) of $60 \%$ at $2 \mathrm{~h}$ after the start of the infusion compared with $27 \%$ in the placebo group (Fig. 2). Thus, intravenous olcegepant was found to be as efficacious as oral sumatriptan in the relief of acute attacks of migraine [42].

Significant superiority over placebo was seen regarding the pain-free rate at $2 \mathrm{~h}$, the sustained pain relief at $24 \mathrm{~h}$ and the improvement of nausea, photophobia and phonophobia (Fig. 3). The response was still rising at $4 \mathrm{~h}$ pointing at a long duration of action. At $24 \mathrm{~h}$ the pain-free rate was better than with triptans, suggesting a lower grade of rebound.

The rate of recurrence was $19 \%$ as compared to a placebo rate of $46 \%$. This is clearly lower than the recurrence rate of triptans which usually is around $28 \%$ [42].

Safety and tolerability studies also showed that olcegepant is well tolerated. Adverse events were, in general, minor and dose-dependent. Transient mild paresthesia was the single most frequent adverse event [60].

One major disadvantage of olcegepant remains in its structure. It is a hydrophilic molecule, has poor penetration across the blood-brain barrier and is not suitable as an oral drug. Considering the crucial advantages of an oral formulation of a drug, a small-molecule, non-peptide CGRP antagonist called MK-0974 was recently developed for the 


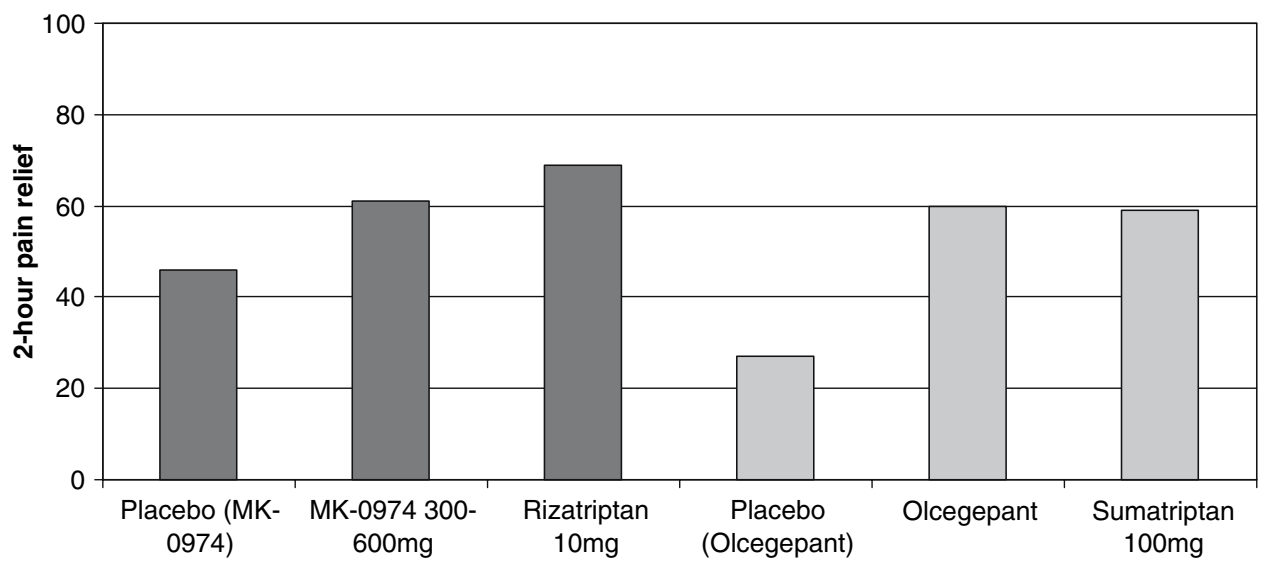

Fig. 2 The anti-migraine effect of the CGRP receptor antagonists $M K$ 0974 and olcegepant and the triptans rizatriptan and sumatriptan compared to placebo at 2-h pain relief. The overall treatment effect of MK-0974 $(p=0.015)$ and rizatriptan $(p=0.010)$ showed significance versus placebo (left part) [69]. A dose of $2.5 \mathrm{mg}$ of olcegepant also showed significant superiority over placebo $(p=0.001)$ [41]. Data of sumatriptan from another study [42] were added to demonstrate the similar efficacy of olcegepant compared to triptans
Fig. 3 Comparison of the sustained pain free-rate at $24 \mathrm{~h}$ of the CGRP receptor antagonists $M K$-0974 and olcegepant, the triptans rizatriptan and sumatriptan, and placebo. $M K$-0974 displayed superior efficacy versus placebo $(p<0.001)$ [69]. In order to compare the efficacy of olcegepant to a triptan, data of sumatriptan from another study [42] were added

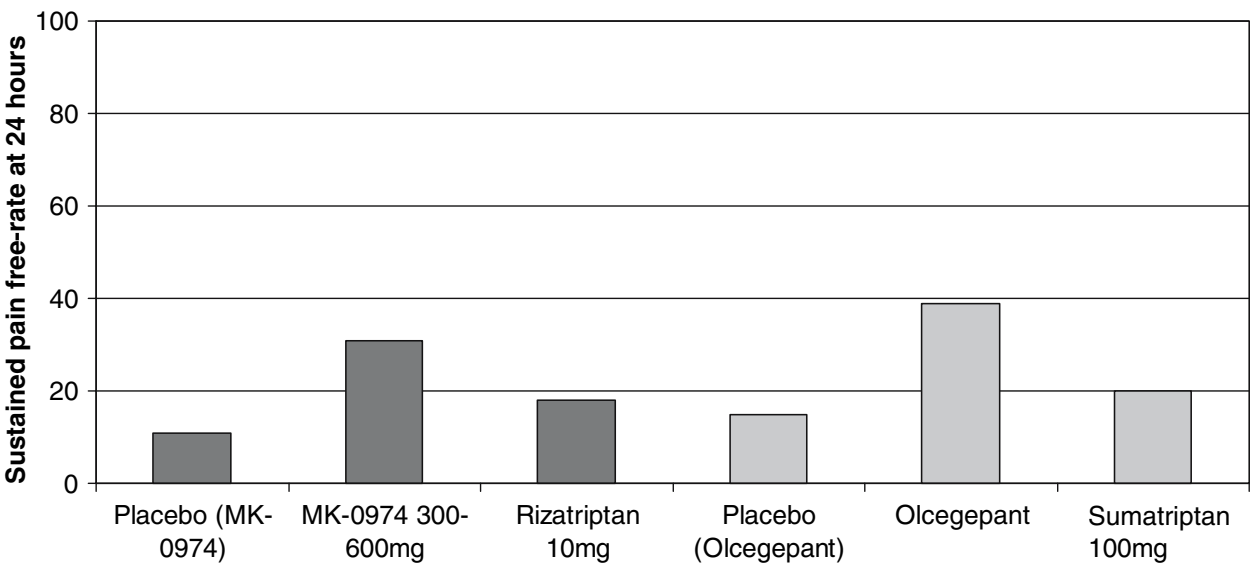

(10 mg) or placebo. As doses below $300 \mathrm{mg}$ were insufficient, they were discontinued. Thus, the overall treatment effect was defined as the average of the 300, 400, and $600 \mathrm{mg}$ doses and showed significance vs. placebo on the primary endpoint ( $p=0.015$ ), which was defined as pain relief at $2 \mathrm{~h}$ after treatment (Fig. 2).

MK-0974 displayed also superior efficacy vs. placebo for secondary endpoints such as pain freedom at $2 \mathrm{~h}$ (overall treatment effect $p<0.001$ ), sustained pain relief at $24 \mathrm{~h}$ (overall treatment effect $p<0.001$ ) or sustained pain freedom at $24 \mathrm{~h}$ (overall treatment effect $p<0.001$ ) (Fig. 3). In addition, MK-0974 provided relief of migraine-associated symptoms such as photophobia, phonophobia and nausea.

Rizatriptan (10 mg) was used as an active control and demonstrated effectiveness vs. placebo $(p=0.010)$ on the primary endpoint defined as pain relief at $2 \mathrm{~h}$. By comparison, 300 and $600 \mathrm{mg}$ doses of MK-0974 appeared to work as well as rizatriptan or showed even numerical superiority regarding the sustained pain freedom at $24 \mathrm{~h}$ and the sustained pain relief at $24 \mathrm{~h}$. 
Concerning tolerability and safety, MK-0974 was generally well-tolerated. The reported side effects were mainly nausea, dizziness and somnolence, but the incidence of adverse experiences seemed to be comparable to the placebo group. In addition, there was no increase in adverse events with increasing dose.

Thus, MK-0974 showed only mild side effects, but significant relief of migraine pain $2 \mathrm{~h}$ after treatment compared to placebo and the relief was sustained through $24 \mathrm{~h}[69]$.

\section{Conclusion}

The novel CGRP receptor antagonists appear to be effective in the treatment of moderate and severe migraine attacks. They do not only provide pain relief at $2 \mathrm{~h}$, but also show efficacy regarding the secondary endpoints such as pain freedom, improvement of associated symptoms and functional disability [41, 69]. In addition, they show a prolonged effect of action compared to the triptans. That may suggest not only less rebound headache but perhaps also a prophylactic possibility. Furthermore, only minor adverse effects were reported and no cardiovascular side effects could be seen so far. This is probably due to the absence of vasoconstrictor properties and it also suggests that vasoconstriction is not necessary to abort acute migraine attacks. This lack of direct vasoconstrictor activity may represent an important advantage over the triptans. Further development of drugs against the CGRP receptor will offer a new promising way of treatment.

Conflicts of interest None.

\section{References}

1. Goadsby PJ (2007) Recent advances in understanding migraine mechanisms, molecules and therapeutics. Trends Mol Med 13(1):39-44

2. Weiller C, May A, Limmroth V et al (1995) Brain stem activation in spontaneous human migraine attacks. Nat Med 1(7):658-660

3. Diener HC, May A (1996) Positron emission tomography studies in acute migraine attacks. In: Sandler M, Ferrari M, Harnett S (eds) Migraine: pharmacology and genetics. Chapman and Hall, London, pp 109-116

4. Afridi SK, Giffin NJ, Kaube $\mathrm{H}$ et al (2005) A positron emission tomographic study in spontaneous migraine. Arch Neurol 62(8):1270-1275

5. Edvinsson L, Jansen Olesen I, Kingman TA et al (1995) Modification of vasoconstrictor responses in cerebral blood vessels by lesioning of the trigeminal nerve: possible involvement of CGRP. Cephalalgia 15(5):373-383

6. McCulloch J, Uddman R, Kingman TA et al (1986) Calcitonin gene-related peptide: functional role in cerebrovascular regulation. Proc Natl Acad Sci U S A 83(15):5731-5735
7. Strong AJ, Fabricius M, Boutelle MG et al (2002) Spreading and synchronous depressions of cortical activity in acutely injured human brain. Stroke 33(12):2738-2743

8. Goadsby PJ, Edvinsson L (1993) The trigeminovascular system and migraine: studies characterizing cerebrovascular and neuropeptide changes seen in humans and cats. Ann Neurol 33(1):4856

9. Edvinsson L, Uddman R (2005) Neurobiology in primary headaches. Brain Res Brain Res Rev 48(3):438-456

10. Tajti J, Uddman R, Moller S et al (1999) Messenger molecules and receptor mRNA in the human trigeminal ganglion. J Auton Nerv Syst 76(2-3):176-183

11. Edvinsson L, Ekman R, Jansen I et al (1987) Calcitonin generelated peptide and cerebral blood vessels: distribution and vasomotor effects. J Cereb Blood Flow Metab 7(6):720-728

12. Gulbenkian S, Uddman R, Edvinsson L (2001) Neuronal messengers in the human cerebral circulation. Peptides 22(6):995-1007

13. Goadsby PJ, Edvinsson L, Ekman R (1990) Vasoactive peptide release in the extracerebral circulation of humans during migraine headache. Ann Neurol 28(2):183-187

14. Juhasz G, Zsombok T, Jakab B et al (2005) Sumatriptan causes parallel decrease in plasma calcitonin gene-related peptide (CGRP) concentration and migraine headache during nitroglycerin induced migraine attack. Cephalalgia 25(3):179-183

15. Juhasz G, Zsombok T, Modos EA et al (2003) NO-induced migraine attack: strong increase in plasma calcitonin gene-related peptide (CGRP) concentration and negative correlation with platelet serotonin release. Pain 106(3):461-470

16. Fanciullacci M, Alessandri M, Figini M et al (1995) Increase in plasma calcitonin gene-related peptide from the extracerebral circulation during nitroglycerin-induced cluster headache attack. Pain 60(2):119-123

17. Longmore J, Razzaque Z, Shaw D et al (1998) Comparison of the vasoconstrictor effects of rizatriptan and sumatriptan in human isolated cranial arteries: immunohistological demonstration of the involvement of 5-HT1B-receptors. $\mathrm{Br} \mathrm{J}$ Clin Pharmacol 46(6):577-582

18. Nilsson T, Longmore J, Shaw D et al (1999) Contractile 5-HT1B receptors in human cerebral arteries: pharmacological characterization and localization with immunocytochemistry. $\mathrm{Br} \mathrm{J}$ Pharmacol 128(6):1133-1140

19. Smith D, Hill RG, Edvinsson L et al (2002) An immunocytochemical investigation of human trigeminal nucleus caudalis: CGRP, substance P and 5-HT1D-receptor immunoreactivities are expressed by trigeminal sensory fibres. Cephalalgia 22(6):424431

20. Hou M, Kanje M, Longmore J et al (2001) 5-HT(1B) and 5HT(1D) receptors in the human trigeminal ganglion: co-localization with calcitonin gene-related peptide, substance $\mathrm{P}$ and nitric oxide synthase. Brain Res 909(1-2):112-120

21. Longmore J, Shaw D, Smith D et al (1997) Differential distribution of 5HT1D- and 5HT1B-immunoreactivity within the human trigemino-cerebrovascular system: implications for the discovery of new antimigraine drugs. Cephalalgia 17(8):833-842

22. Martin GR (1997) Pre-clinical pharmacology of zolmitriptan (Zomig; formerly 311C90), a centrally and peripherally acting 5HT1B/1D agonist for migraine. Cephalalgia 17(Suppl 18):4-14

23. Tfelt-Hansen P (2007) Parenteral vs. oral sumatriptan and naratriptan: plasma levels and efficacy in migraine. A comment. J Headache Pain 8:287-290

24. Tfelt-Hansen P, Young WB, Silberstein SD (2006) Antiemetics, prokinetics, neuroleptic and miscellaneous drugs in the acute treatment of migraine. In: Olesen J, Goadsby PJ, Ramadan NM, Tfelt-Hansen P, Welch KMA (eds) The headaches, 3rd edn. Lippincott Williams and Wilkins, Philadelphia, pp 505-513 
25. Volans GN (1974) Absorption of effervescent aspirin during migraine. Br Med J 4(5939):265-268

26. Edvinsson L, Uddman E, Wackenfors A et al (2005) Triptaninduced contractile (5-HT1B receptor) responses in human cerebral and coronary arteries: relationship to clinical effect. Clin Sci (Lond) 109(3):335-342

27. Nilsson T, Longmore J, Shaw D et al (1999) Characterisation of 5 -HT receptors in human coronary arteries by molecular and pharmacological techniques. Eur J Pharmacol 372(1):49-56

28. MaassenVanDenBrink A, van den Broek RW, de Vries R et al (2000) Craniovascular selectivity of eletriptan and sumatriptan in human isolated blood vessels. Neurology 55(10):1524-1530

29. MaassenVanDenBrink A, Reekers M, Bax WA et al (1998) Coronary side-effect potential of current and prospective antimigraine drugs. Circulation 98(1):25-30

30. Goadsby PJ (2005) Migraine, allodynia, sensitisation and all of that. Eur Neurol 53(Suppl 1):10-16

31. Burstein R, Yarnitsky D, Goor-Aryeh I et al (2000) An association between migraine and cutaneous allodynia. Ann Neurol 47(5):614-624

32. Bolay H, Reuter U, Dunn AK et al (2002) Intrinsic brain activity triggers trigeminal meningeal afferents in a migraine model. Nat Med 8(2):136-142

33. Lovati C, D'Amico D, Rosa S et al (2007) Allodynia in different forms of migraine. Neurol Sci 28(Suppl 2):S220-221

34. Burstein R, Jakubowski M (2004) Analgesic triptan action in an animal model of intracranial pain: a race against the development of central sensitization. Ann Neurol 55(1):27-36

35. Levy D, Jakubowski M, Burstein R (2004) Disruption of communication between peripheral and central trigeminovascular neurons mediates the antimigraine action of 5HT 1B/1D receptor agonists. Proc Natl Acad Sci U S A 101(12):4274-4279

36. Mathew NT, Kailasam J, Seifert T (2004) Clinical recognition of allodynia in migraine. Neurology 63(5):848-852

37. Linde M, Mellberg A, Dahlof C (2006) Subcutaneous sumatriptan provides symptomatic relief at any pain intensity or time during the migraine attack. Cephalalgia 26(2):113-121

38. Edvinsson L, Jansen I, Kingman TA et al (1990) Cerebrovascular responses to capsaicin in vitro and in situ. $\mathrm{Br} \mathrm{J}$ Pharmacol 100(2):312-318

39. Jansen-Olesen I, Mortensen A, Edvinsson L (1996) Calcitonin gene-related peptide is released from capsaicin-sensitive nerve fibres and induces vasodilatation of human cerebral arteries concomitant with activation of adenylyl cyclase. Cephalalgia 16(5):310-316

40. Petersen KA, Birk S, Doods $\mathrm{H}$ et al (2004) Inhibitory effect of BIBN4096BS on cephalic vasodilatation induced by CGRP or transcranial electrical stimulation in the rat. $\mathrm{Br} \mathrm{J}$ Pharmacol 143(6):697-704

41. Olesen J, Diener HC, Husstedt IW et al (2004) Calcitonin generelated peptide receptor antagonist BIBN 4096 BS for the acute treatment of migraine. N Engl J Med 350(11):1104-1110

42. Ferrari MD, Roon KI, Lipton RB et al (2001) Oral triptans (serotonin 5-HT(1B/1D) agonists) in acute migraine treatment: a meta-analysis of 53 trials. Lancet 358(9294):1668-1675

43. Oliver KR, Wainwright A, Edvinsson L et al (2002) Immunohistochemical localization of calcitonin receptor-like receptor and receptor activity-modifying proteins in the human cerebral vasculature. J Cereb Blood Flow Metab 22(5):620-629

44. Edvinsson L, Alm R, Shaw D et al (2002) Effect of the CGRP receptor antagonist BIBN4096BS in human cerebral, coronary and omental arteries and in SK-N-MC cells. Eur J Pharmacol 434(1-2):49-53

45. Petersen KA, Nilsson E, Olesen J et al (2005) Presence and function of the calcitonin gene-related peptide receptor on rat pial arteries investigated in vitro and in vivo. Cephalalgia 25(6):424 432

46. Storer RJ, Akerman S, Goadsby PJ (2004) Calcitonin gene-related peptide (CGRP) modulates nociceptive trigeminovascular transmission in the cat. Br J Pharmacol 142(7):1171-1181

47. Zhang Z, Winborn CS, Marquez de Prado B et al (2007) Sensitization of calcitonin gene-related peptide receptors by receptor activity-modifying protein-1 in the trigeminal ganglion. $\mathrm{J}$ Neurosci 27(10):2693-2703

48. McLatchie LM, Fraser NJ, Main MJ et al (1998) RAMPs regulate the transport and ligand specificity of the calcitonin-receptor-like receptor. Nature 393(6683):333-339

49. Foord SM, Marshall FH (1999) RAMPs: accessory proteins for seven transmembrane domain receptors. Trends Pharmacol Sci 20(5):184-187

50. Hay DL, Poyner DR, Sexton PM (2006) GPCR modulation by RAMPs. Pharmacol Ther 109(1-2):173-197

51. Evans BN, Rosenblatt MI, Mnayer LO et al (2000) CGRP-RCP, a novel protein required for signal transduction at calcitonin generelated peptide and adrenomedullin receptors. J Biol Chem 275(40):31438-31443

52. Goadsby PJ (2005) Calcitonin gene-related peptide antagonists as treatments of migraine and other primary headaches. Drugs 65(18):2557-2567

53. Rudolf K, Eberlein W, Engel W et al (2005) Development of human calcitonin gene-related peptide (CGRP) receptor antagonists. 1. Potent and selective small molecule CGRP antagonists. 1-[N2-[3,5-dibromo- $N$-[[4-(3,4-dihydro-2(1H)-oxoquinazolin-3yl)-1-piperidi nyl]carbonyl]-D-tyrosyl]-1-lysyl]-4-(4-pyridinyl)piperazine: the first CGRP antagonist for clinical trials in acute migraine. J Med Chem 48(19):5921-5931

54. Doods H, Hallermayer G, Wu D et al (2000) Pharmacological profile of BIBN4096BS, the first selective small molecule CGRP antagonist. Br J Pharmacol 129(3):420-423

55. Gupta S, Mehrotra S, Avezaat CJ et al (2006) Characterisation of CGRP receptors in the human isolated middle meningeal artery. Life Sci 79(3):265-271

56. Jansen-Olesen I, Jorgensen L, Engel U et al (2003) In-depth characterization of CGRP receptors in human intracranial arteries. Eur J Pharmacol 481(2-3):207-216

57. Wimalawansa SJ (1996) Calcitonin gene-related peptide and its receptors: molecular genetics, physiology, pathophysiology, and therapeutic potentials. Endocr Rev 17(5):533-585

58. Saetrum Opgaard O, Hasbak P, de Vries R et al (2000) Positive inotropy mediated via CGRP receptors in isolated human myocardial trabeculae. Eur J Pharmacol 397(2-3):373-382

59. Wallengren J, Ekman R, Sundler F (1987) Occurrence and distribution of neuropeptides in the human skin. An immunocytochemical and immunochemical study on normal skin and blister fluid from inflamed skin. Acta Derm Venereol 67(3):185-192

60. Iovino M, Feifel U, Yong CL et al (2004) Safety, tolerability and pharmacokinetics of BIBN 4096 BS, the first selective small molecule calcitonin gene-related peptide receptor antagonist, following single intravenous administration in healthy volunteers. Cephalalgia 24(8):645-656

61. Edvinsson L (2002) Calcitonin gene-related peptide (CGRP) in cerebrovascular disease. ScientificWorldJournal 2:1484-1490

62. Petersen KA, Birk S, Lassen LH et al (2005) The CGRP-antagonist, BIBN4096BS does not affect cerebral or systemic haemodynamics in healthy volunteers. Cephalalgia 25(2):139-147

63. Gupta S, Akerman S, van den Maagdenberg AM et al (2006) Intravital microscopy on a closed cranial window in mice: a model to study trigeminovascular mechanisms involved in migraine. Cephalalgia 26(11):1294-1303 
64. Edvinsson L (2004) Blockade of CGRP receptors in the intracranial vasculature: a new target in the treatment of headache. Cephalalgia 24(8):611-622

65. Petersen KA, Lassen LH, Birk S et al (2005) BIBN4096BS antagonizes human alpha-calcitonin gene related peptide-induced headache and extracerebral artery dilatation. Clin Pharmacol Ther 77(3):202-213

66. Edvinsson L, Nilsson E, Jansen-Olesen I (2007) Inhibitory effect of BIBN4096BS, CGRP(8-37), a CGRP antibody and an RNASpiegelmer on CGRP induced vasodilatation in the perfused and non-perfused rat middle cerebral artery. $\mathrm{Br} \mathrm{J}$ Pharmacol 150(5):633-640

67. Paone DV, Shaw AW, Nguyen DN et al (2007) Potent, orally bioavailable calcitonin gene-related peptide receptor antagonists for the treatment of migraine: discovery of $N$-[(3R,6S)-6-(2,3Difluorophenyl)-2-oxo-1- (2,2,2-trifluoroethyl)azepan-3-yl]-4(2-oxo-2,3-dihydro-1H-imidazo[4,5-b]pyridin-1-yl)piperidine-1carboxamide (MK-0974). J Med Chem 50(23):5564-5567

68. Hershey JC, Corcoran HA, Baskin EP et al (2005) Investigation of the species selectivity of a nonpeptide CGRP receptor antagonist using a novel pharmacodynamic assay. Regul Pept 127(13):71-77

69. Ho TW, Mannix LK, Fan X et al (2007) Randomized controlled trial of an oral CGRP antagonist, MK-0974, in acute treatment of migraine. Neurology (Epub ahead of print) 